\title{
M2 to D2 and vice versa by 3-Lie and Lie bialgebra
}

\author{
M. Aali-Javanangrouh ${ }^{\mathrm{a}}$, A. Rezaei-Aghdam ${ }^{\mathrm{b}}$ \\ Department of Physics, Faculty of science, Azarbaijan Shahid Madani University, 53714-161 Tabriz, Iran
}

Received: 30 August 2016 / Accepted: 1 November 2016 / Published online: 19 November 2016

(c) The Author(s) 2016. This article is published with open access at Springerlink.com

\begin{abstract}
Using the concept of a 3-Lie bialgebra, which has recently been defined in arXiv:1604.04475, we construct a Bagger-Lambert-Gustavson (BLG) model for the M2-brane on a Manin triple of a special 3-Lie bialgebra. Then by using the correspondence and the relation between those 3-Lie bialgebra with Lie bialgebra, we reduce this model to an $N=(4,4)$ WZW model (D2-brane), such that its algebraic structure is a Lie bialgebra with one 2-cocycle. In this manner by using the correspondence of the 3-Lie bialgebra and Lie bialgebra (for this special 3-Lie algebra) one can construct the M2-brane from a D2-brane and vice versa.
\end{abstract}

\section{Introduction}

M-theory is a magical theory of which quite little is known, so to improve its current definition we must focus on 11 dimensional (11d) supergravity $[1,2]$. These supergravity theories are low-energy limits of M-theory, whereas 10d supergravity is the low-energy limit of superstring. Therefore, there should be a connection between M-theory and string theory like existing connection between $11 \mathrm{~d}$ and $10 \mathrm{~d}$ supergravity. If we could make this connection clear, then a lot of unknown issues as regards M-theory would be resolved. Our knowledge of M-theory has been achieved by comparing and groping with D-branes [3-5]. Many attempts have been made to obtain the effective action for multiple M2-brane, the most important of which are cited in [6-11]. Basu and Harvey [12] applied a 3-bracket in the BPS equation in order to explain N coincident M2-branes ending on the M5-brane. This equation has been given by comprising the Nahm equation for string theory [13]. Bagger and Lambert [6-8] and Gustavsson [9] independently wrote the transformation of the fields for the M2-brane according to the D2-brane. They obtained the equation of motion of fields by using the closure

\footnotetext{
a e-mail: aali@ azaruniv.edu

be-mail: rezaei-a@azaruniv.edu
}

of the supersymmetric transformation algebra, and writing a Lagrangian in such a way that its equations of motion are the same. The Bagger-Lambert-Gustavsson (BLG) model [69] has a Lagrangian with maximal supersymmetry $(N=8)$ for the description of two M2-branes [14], which use the 3Lie algebra. As the earlier example, the algebra $A_{4}$ was the only known non-trivial 3-Lie algebra [8]. Mukhi and Papageorgakis $[15,16]$ were able to convert the topological term (Chern-Simon term) to the dynamical one, i.e., the YangMills term, by assigning a vacuum expectation value to a scalar fields of the BLG Lagrangian and using the Higgs mechanism. On the other hand, if one dualizes M-theory on a circle, one can obtain type IIA string theory (D2-brane) [17]. This can be considered as a trick for going from M2 to D2, as was performed for $A_{4}$. Consequently, a 3-Lie algebra was constructed from an arbitrary Lie algebra [18] and the BLG model on this 3-Lie algebras were studied in later works [19-23]. In all this works one can obtain the M2-model going to D2 but these are not standard methods of construction of going from M2 from D2. Here we will try to perform another method using the concept of the 3-Lie bialgebra [24].

Lie bialgebras [25] are algebraic structures of PoissonLie groups [26], which play an important role in the theory of classical integrable systems (see [27] for a review). They also play an important role in $N=(2,2)$ and $N=(4,4)$ supersymmetric WZW models [28-30]. In Ref. [31] we have studied the algebraic structure of $N=(2,2)$ and $N=(4,4)$ supersymmetric WZW models in more detail. The concept of 3-Lie algebra was described in Fillipov's work for the first time [32] following the pioneering work of Nambu in a different formulation of classical mechanics [33]. In Ref. [24] we define the concept of the 3-Lie bialgebra by using the cohomology of 3-Lie algebras. We believe that the introduction of the 3-Lie bialgebras can play an important role in M-theory. In this paper we will follow some steps in this direction. We will express the BLG Lagrangian on the Manin triple of a special 3-Lie bialgebra and use a similar procedure applied by Mukhi and Papageorgakis [15,16], in obtaining 
the Yang-Mills in addition to other terms which are those of WZW. The extra term is the square of the B-field for WZW models [34-36]. If the space-time coordinates were algebraic indices (like space-time coordinates, i.e., scalar and fermion fields in the BLG model) then the B-field of the $N=(4,4)$ WZW model could be obtained from this form. In this way if one knows about $N=(4,4)$ WZW models in detail, then one can obtain information about BLG and vice versa, i.e., one can construct the M2-model from the D2-model and vice versa.

The outline of the paper is as follows. We review BLG action and the correspondence between M-theory and string theory in section two. In Sect. 3 we review the definition of the 3-Lie bialgebra [24] and give an example of a one-toone correspondence between 3-Lie bialgebra and Lie bialgebra. Consequently, in Sect. 4 we express BLG model (M2model) on the Manin triple of that 3-Lie bialgebra and show that it turns into Yang-Mills and $N=(4,4)$ WZW model (D2-model). In this manner we show that using the correspondence of the 3-Lie bialgebra and Lie bialgebra one can construct M2-model from D2 and vice versa. For a further description of the obtained model; in Sect. 5 we show that the WZW model can be obtained from a DBI action with extra Lie algebra valued fields.

\section{BLG model}

Here for self consistency of the paper and presentation of the notation, we give a short review of the BLG model. The multiple M2-brane model of Bagger-Lambert [6-8] and Gustavsson [9] (BLG) is based on the 3-Lie algebra. In this algebra the Lie bracket is generalized to the 3-Lie bracket [32]. The n-Lie algebra was introduced by Filippov in 1985 [32] as an extension of the Nambu bracket [33] to Lie algebras. 3-Lie algebras are a special kind of n-Lie algebras, and they have many applications in mathematical and theoretical physics [6-9]. The 3-Lie algebra $\mathcal{A}[32,37]$ with the basis $\left\{T^{a}\right\}$ is a vector space $\mathcal{A}$, which is endowed with the following three antisymmetric brackets:

$\left[T^{a}, T^{b}, T^{c}\right]=f_{d}^{a b c} T^{d}, \quad a, b, c, d=1, \ldots, \operatorname{dim} \mathcal{A}$,

so as to satisfy the following fundamental identity [32]

$$
\begin{aligned}
{\left[T^{g}, T^{d},\left[T^{a}, T^{b}, T^{c}\right]\right]=} & {\left[\left[T^{g}, T^{d}, T^{a}\right], T^{b}, T^{c}\right] } \\
& +\left[T^{a},\left[T^{g}, T^{d}, T^{b}\right], T^{c}\right] \\
& +\left[T^{a}, T^{b},\left[T^{g}, T^{d}, T^{c}\right]\right],
\end{aligned}
$$

where it can be redefined by the structure constant of $\mathcal{A}\left(f_{d}^{a b c}\right)$ in the following form:

$$
f_{e}^{a b c} f_{f}^{g d e}-f_{e}^{g d a} f_{f}^{e b c}-f_{e}^{g d b} f_{f}^{a e c}-f_{e}^{g d c} f_{f}^{a b e}=0 .
$$

For the BLG model the following supersymmetric (SUSY) transformations are proposed [6-8]:

$$
\begin{aligned}
\delta X_{a}^{I} & =i \bar{\epsilon} \Gamma^{I} \Psi_{a}, \\
\delta \Psi_{a} & =D_{\mu} X_{a}^{I} \Gamma^{\mu} \Gamma_{I} \epsilon-\frac{1}{2} X_{b}^{I} X_{c}^{J} X_{d}^{K} f_{a}^{b c d} \Gamma_{I J K} \epsilon, \\
\delta\left(\hat{A}_{\mu}\right)_{b}^{a} & =i \bar{\epsilon} \Gamma_{\mu} \Gamma_{I} X_{c}^{I} \Psi_{d} f_{b}^{c d a},
\end{aligned}
$$

where $\left(\hat{A}_{v}\right)_{a}^{b}=f_{a}^{c d b} A_{v c d}$, and the indices $I, J, \ldots=$ $1,2, \ldots, 8$ apply for transverse coordinates with $S O(8)$ symmetry (R-symmetry); and the indices $\mu, v, \ldots=0,1,2$ indicate the world volume coordinate with the symmetry $S O(1,2) .{ }^{1}$ Also, $\Gamma_{I}$ s are Dirac matrices and $X^{I}$ are the transverse coordinates of the 3 -Lie algebra valued coordinates and $\Psi$ is a 16 component Majorana spinor of a 3-Lie algebra valued, conforming the chirality condition using the following relation:

$\Gamma^{012} \Psi=-\Psi$

such that for the supersymmetric parameter $\epsilon$ we have

$\Gamma^{012} \epsilon=\epsilon$

and the covariant derivative $D_{\mu}$ has the form

$D_{\mu} X_{a}^{(I)}=\partial_{\mu} X_{a}^{(I)}+f_{a}^{b c d} A_{\mu c d} X_{b}^{(I)}$

Furthermore, the $\Gamma_{I J}$ and $\Gamma_{I J K}$ have the following forms:

$\Gamma^{I J}=\frac{1}{2}\left(\Gamma^{I} \Gamma^{J}-\Gamma^{J} \Gamma^{I}\right)$,

$\left\{\Gamma^{I}, \Gamma_{J K L}\right\}=6 \delta_{[J}^{I} \Gamma_{K L]}$.

Using the assumption that the algebra of the SUSY transformations (4) must be closed, the following relations can be realized [6]:

$$
\begin{aligned}
& \Gamma^{\mu} D_{\mu} \Psi_{a}+\frac{1}{2} \Gamma_{I J} X_{c}^{I} X_{d}^{J} \Psi_{b} f_{a}^{c d b}=0, \\
& D^{2} X_{a}^{I}-\frac{i}{2} \tilde{\Psi}_{c} \Gamma_{J}^{I} X_{d}^{J} \Psi_{b} f_{a}^{c d b} \\
& \quad+\frac{1}{2} f_{a}^{b c d} f_{d}^{e f g} X_{b}^{J} X_{c}^{K} X_{e}^{I} X_{f}^{J} X_{g}^{K}=0, \\
& \left(\hat{F}_{\mu \nu}\right)_{a}^{b}+\epsilon_{\mu \nu \lambda}\left(X_{c}^{J} D^{\lambda} X_{d}^{J}+\frac{i}{2} \bar{\Psi}_{c} \Gamma^{\lambda} \Psi_{d}\right) f_{a}^{c d b}=0,
\end{aligned}
$$

where

$$
\begin{aligned}
\left(\hat{F}_{\mu \nu}\right)_{a}^{b}= & \partial_{\mu}\left(\hat{A_{\nu}}\right)_{a}^{b}-\partial_{\nu}\left(\hat{A_{\mu}}\right)_{a}^{b} \\
& +\left(\hat{A}_{\mu}\right)_{c}^{b}\left(\hat{A}_{\nu}\right)_{a}^{c}-\left(\hat{A}_{\nu}\right)_{c}^{b}\left(\hat{A}_{\mu}\right)_{a}^{c},
\end{aligned}
$$

\footnotetext{
1 The presence of the M2-brane breaks the Lorentz invariance $S O(1,10)$ to $S O(1,2) \times S O(8)$.
} 
and $D^{2}=D_{\mu} D^{\mu}$. Similarly Bagger and Lambert have proposed the following Lagrangian [7] such that Eqs. (10)-(12) are its equations of motion:

$$
\begin{aligned}
L= & -\frac{1}{2} D_{\mu} X^{a(I)} D^{\mu} X_{a}^{(I)} \\
& +\frac{i}{2} \bar{\psi}^{a} \Gamma^{\mu} D_{\mu} \psi_{a}+\frac{i}{4} f^{a b c d} \bar{\psi}_{b} \Gamma^{I J} X_{c(I)} X_{d(J)} \psi_{a} \\
& -\frac{1}{12} f^{a b c d} f^{e f g}{ }_{d} X_{a}^{(I)} X_{b}^{(J)} X_{c}^{(K)} X_{e}^{(I)} X_{f}^{(J)} X_{g}^{(K)} \\
& +\frac{1}{2} \epsilon^{\mu \nu \lambda}\left[f^{a b c d} A_{\mu a b} \partial_{\nu} A_{\lambda c d}\right. \\
& \left.+\frac{2}{3} f^{c d a}{ }_{g} f^{e f g b} A_{\mu a b} A_{\nu c d} A_{\lambda e f}\right] .
\end{aligned}
$$

The above Lagrangian is invariant under SUSY transformation (4). In order for the degrees of Fermion and Boson not to vary in Lagrangian, one must use topological term which is the Chern-Simon term (the fifth term in the bracket at the above Lagrangian) [38]:

$L_{\mathrm{CS}}=\operatorname{Tr}\left(\epsilon^{\mu \nu \lambda}\left(A_{\mu} \partial_{\nu} A_{\lambda}+\frac{2 i}{3}\left(A_{\mu} A_{\nu} A_{\lambda}\right)\right)\right)$,

with

$$
F_{\mu \nu}=\partial_{\mu} A_{\nu}-\partial_{\nu} A_{\mu}+i\left[A_{\mu}, A_{\nu}\right],
$$

which is equipped with the 3-Lie algebra. Now, we will try to extract the BLG model from a superstring model (D2) and vice versa. In our perspective for this propose we need to apply the concept of 3-Lie bialgebra. The definition of the 3Lie bialgebra is given in [24], however, for self containment of the paper we give a short review of this concept in the following section.

\section{3-Lie bialgebra}

In this section we review the definitions of the 3-Lie bialgebra.

Definition: A Lie algebra $\mathcal{G}$ with co-commutator $\delta: \mathcal{G} \rightarrow$ $\mathcal{G} \otimes \mathcal{G}$ is a Lie bialgebra if [27]:

(a) $\delta$ is a one-cocycle, i.e.,

$$
\delta\left(\left[T^{i}, T^{j}\right]\right)=a d^{(2)} T^{j} \delta\left(T^{i}\right)-a d^{(2)} T^{i} \delta\left(T^{j}\right),
$$

where

$a d^{(2)} T^{j}=a d_{T^{j}} \otimes 1+1 \otimes a d_{T^{j}}$,

and $\left\{T^{i}\right\} \mathrm{s}$ are bases for the Lie algebra $\mathcal{G}$ (here 1 is an identity map on $\mathcal{G}$ ), (b) the dual map ${ }^{t} \delta: \mathcal{G}^{*} \otimes \mathcal{G}^{*} \rightarrow \mathcal{G}^{*}$ is a commutator on $\mathcal{G}^{*}$ (dual space of $\mathcal{G}$ ) as in the following definition:

$$
\left(\tilde{T}_{i} \otimes \tilde{T}_{j}, \delta\left(T^{k}\right)\right)=\left({ }^{t} \delta\left(\tilde{T}_{i} \otimes \tilde{T}_{j}\right), T^{k}\right)=\left(\left[\tilde{T}_{i}, \tilde{T}_{j}\right], T^{k}\right),
$$

where $\left\{\tilde{T}_{i}\right\}$ is the base for the space $\mathcal{G}^{*}$ and (, ) is the pairing between $\mathcal{G}$ and $\mathcal{G}^{*}$. In this way there is a Lie algebra structure on the space $\mathcal{G}^{*}$. The Lie bialgebra is shown with $(\mathcal{G}, \delta)$ or $\left(\mathcal{G}, \mathcal{G}^{*}\right)$.

Definition: $\left(\mathcal{D}, \mathcal{G}, \mathcal{G}^{*}\right)$ is a Manin triple, the triple of Lie algebras $\mathcal{D}, \mathcal{G}$ and $\mathcal{G}^{*}$, such that there is a nondegenerate, symmetric and ad-invariant inner product on $\mathcal{D}$ with the following properties [27]:

(a) $\mathcal{G}$ and $\mathcal{G}^{*}$ are subalgebras of $\mathcal{D}$.

(b) $\mathcal{D}=\mathcal{G} \oplus \mathcal{G}^{*}$ as a vector space.

(c) $\mathcal{G}$ and $\mathcal{G}^{*}$ are isotropic, i.e.,

$$
\left(T^{i}, \tilde{T}_{j}\right)=\delta_{j}^{i}, \quad\left(T^{i}, T^{j}\right)=\left(\tilde{T}_{i}, \tilde{T}_{j}\right)=0 .
$$

The Jacobi identity for $\mathcal{D}=\mathcal{G} \oplus \mathcal{G}^{*}$ results in the following identities [39]:

$f_{k}^{i j} f_{m}^{k l}-f_{m}^{i k} f_{k}^{j l}+f_{m}^{j k} f_{k}^{i l}=0$,

$\tilde{f}_{i j}^{k} \tilde{f}_{k l}^{m}-\tilde{f}_{i k}^{m} \tilde{f}_{j l}^{k}+\tilde{f}_{j k}^{m} \tilde{f}_{i l}^{k}=0$,

$-f_{k}^{i j} \tilde{f}_{l m}^{k}+f_{l}^{i k} \tilde{f}_{k m}^{j}-f_{m}^{j k} \tilde{f}_{l k}^{i}-f_{l}^{j k} \tilde{f}_{k m}^{i}+f_{m}^{i k} \tilde{f}_{l k}^{j}=0$,

where $f_{k}^{i j}$ and $\tilde{f}_{i j}^{k}$ are the structure constants of the Lie algebras $\mathcal{G}$ and $\mathcal{G}^{*}$, respectively (i.e. $\left[T^{i}, T^{j}\right]=f_{k}^{i j} T_{k}$, $\left[\tilde{T}_{i}, \tilde{T}_{j}\right]=\tilde{f}_{i j}^{k} \tilde{T}_{k}$ ). Note that (20) and (21) are Jacobi identities for the Lie algebras $\mathcal{G}$ and $\mathcal{G}^{*}$, respectively, and (22) is the mix Jacobi identity on $\mathcal{D}$.

Theorem: There exists a one-to-one correspondence between Lie bialgebra $\left(\mathcal{G}, \mathcal{G}^{*}\right)$ and the Manin triple $\left(\mathcal{D}, \mathcal{G}, \mathcal{G}^{*}\right)$ [27].

We have defined the 3-Lie bialgebra in [24] as follows.

Definition: A 3-Lie algebra $\mathcal{A}$ with co-commutator $\delta: \mathcal{A} \rightarrow$ $\mathcal{A} \otimes \mathcal{A} \otimes \mathcal{A}$ is a 3-Lie bialgebra if [24]:

(a) $\delta$ is a one-cocycle of $\mathcal{A}$ with value in $\otimes^{3} \mathcal{A}$, i.e:

$$
\begin{aligned}
\delta\left(\left[T^{a}, T^{b}, T^{c}\right]\right)= & a d^{(3)} T^{b} \otimes T^{c} \delta\left(T^{a}\right)-a d^{(3)} T^{a} \otimes T^{c} \delta\left(T^{b}\right) \\
& +a d^{(3)} T^{a} \otimes T^{b} \delta\left(T^{c}\right),
\end{aligned}
$$


such that

$$
\begin{aligned}
a d^{(3)} T^{b} \otimes T^{c} & =a d_{T^{b} \otimes T^{c}} \otimes 1 \otimes 1+1 \otimes a d_{T^{b} \otimes T^{c}} \otimes 1 \\
& +1 \otimes 1 \otimes a d_{T^{b} \otimes T^{c}},
\end{aligned}
$$

where $\left\{T^{a}\right\}$ s are bases of the 3-Lie algebra $\mathcal{A}$ and we have $a d_{T^{a} \otimes T^{b}} T^{c}=\left[T^{a}, T^{b}, T^{c}\right][40]$.

(b) The dual map ${ }^{t} \delta: \otimes^{3} \mathcal{A}^{*} \rightarrow \mathcal{A}^{*}$ is a 3-Lie bracket on $\mathcal{A}^{*}$ (dual space of $\mathcal{A}$ ), which is a commutator on $\mathcal{G}^{*}$ satisfying the fundamental identity

$$
\begin{aligned}
\left(\tilde{T}_{a} \otimes \tilde{T}_{b} \otimes \tilde{T}_{c}, \delta\left(T^{d}\right)\right) & =\left({ }^{t} \delta\left(\tilde{T}_{a} \otimes \tilde{T}_{b} \otimes \tilde{T}_{c}\right), T^{d}\right) \\
& =\left(\left[\tilde{T}_{a}, \tilde{T}_{b}, \tilde{T}_{c}\right], T^{d}\right),
\end{aligned}
$$

in which $\left\{\tilde{T}_{a}\right\}$ is the base for the space $\mathcal{A}^{*}$ and (, ) is a natural pairing between $\mathcal{A}$ and $\mathcal{A}^{*}$. In this way by $\mathcal{A}^{*}$ one constructs a 3-Lie algebra. The 3-Lie bialgebra can be denoted either by $\left(\mathcal{A}, \mathcal{A}^{*}\right)$ or $(\mathcal{A}, \delta)$.

Definition: $\left(\mathcal{D}, \mathcal{A}, \mathcal{A}^{*}\right)$ is a Manin triple, a triple of 3-Lie algebras $\mathcal{D}, \mathcal{A}$ and $\mathcal{A}^{*}$ such that there is a nondegenerate, symmetric and ad-invariant inner product on $\mathcal{D}$ with the following properties $[24]:^{2}$

(a) $\mathcal{A}$ and $\mathcal{A}^{*}$ are 3-Lie subalgebras of $\mathcal{D}$,

(b) $\mathcal{D}=\mathcal{A} \oplus \mathcal{A}^{*}$ as a vector space,

(c) $\mathcal{A}$ and $\mathcal{A}^{*}$ are isotropic, i.e.

$\left(T^{a}, \tilde{T}_{b}\right)=\delta_{b}^{a},\left(T^{a}, T^{b}\right)=\left(\tilde{T}_{a}, \tilde{T}_{b}\right)=0$.

By using the fundamental identity (2), Eq. (23) and the relation $\delta\left(T^{a}\right)=\tilde{f}_{b c d}^{a} T^{b} \otimes T^{c} \otimes T^{d}$, one can obtain the following fundamental and mix fundamental identities [24]:

$$
\begin{aligned}
& f_{g}^{a e f} f^{b c d g}-f_{g}^{b e f} f^{a c d g}+f_{g}^{c e f} f^{a b d g}-f_{g}^{d e f} f^{a b c g}=0, \\
& \tilde{f}_{a e f}^{g} \tilde{f}_{b c d g}-\tilde{f}_{b e f}^{g} \tilde{f}_{a c d g}+\tilde{f}_{c e f}^{g} \tilde{f}_{a b d g}-\tilde{f}_{d e f}^{g} \tilde{f}_{a b c g}=0 \\
& f^{a b c} \tilde{f}_{d e f}{ }^{g}=f_{f}^{g b c} \tilde{f}_{d e g}{ }^{a}+f^{g b c}{ }_{e} \tilde{f}_{d f g}{ }^{a} \\
& -f^{g b c}{ }_{d} \tilde{f}_{e f g}{ }^{a}-f^{g a c}{ }_{f} \tilde{f}_{d e g}{ }^{b}+f^{g a c}{ }_{e} \tilde{f}_{d f g}{ }^{b} \\
& -f^{g a c}{ }_{d} \tilde{f}_{e f g}{ }^{b}+f^{g a b}{ }_{f} \tilde{f}_{d e g}{ }^{c}{ }^{c} \\
& -f^{g a b}{ }_{e} \tilde{f}_{d f g}{ }^{c}+f^{g a b}{ }_{d} \tilde{f}_{e f g}{ }^{c},
\end{aligned}
$$

where $f_{d}^{a b c}$ and $\tilde{f}_{a b c}^{d}$ are the structure constants of the 3-Lie algebras $\mathcal{A}$ and $\mathcal{A}^{*}$, respectively.

\footnotetext{
2 Note that in general the vector space $\mathcal{D}$ is not a 3-Lie algebra.
}

\subsection{An example}

Now, we will consider a special example of a 3-Lie bialgebra related to the 3-Lie algebra $\mathcal{A}_{\mathcal{G}}$ and Lie algebra $\mathcal{G}$. ${ }^{3}$ The 3Lie algebras $\mathcal{A}_{\mathcal{G}}$ (mentioned in [18] for the first time) have commutation relations as follows:

$$
\begin{aligned}
{\left[T^{-}, T^{a}, T^{b}\right] } & =0, \\
{\left[T^{+}, T^{i}, T^{j}\right] } & =f_{k}^{i j} T^{k}, \\
{\left[T^{i}, T^{j}, T^{k}\right] } & =f^{i j k} T^{-},
\end{aligned}
$$

where the $\left\{T^{i}\right\}$ are a basis of the Lie algebra $\mathcal{G}\left(\left[T^{i}, T^{j}\right]=\right.$ $f_{k}^{i j} T^{k}$ with $\left.i, j, k=1,2, \ldots, \operatorname{dim} \mathcal{G}\right)$ and $f_{k}^{i j}$ is its structure constant. ${ }^{4}$ Furthermore, $T^{-}$and $T^{+}$are new generators and we have $a=+,-, i$. Now we propose that there exists a 3-Lie algebra structure on $\mathcal{A}_{\mathcal{G}^{*}}^{*}$ with similar commutation relations:

$$
\begin{aligned}
{\left[\tilde{T}_{-}, \tilde{T}_{a}, \tilde{T}_{b}\right] } & =0, \\
{\left[\tilde{T}_{+}, \tilde{T}_{i}, \tilde{T}_{j}\right] } & =\tilde{f}_{i j}^{k} \tilde{T}_{k}, \\
{\left[\tilde{T}_{i}, \tilde{T}_{j}, \tilde{T}_{k}\right] } & =\tilde{f}_{i j k} T_{-},
\end{aligned}
$$

such that $\mathcal{G}^{*}\left(\left[\tilde{T}_{i}, \tilde{T}_{j}\right]=\tilde{f}_{i j}^{k} \tilde{T}^{k}\right.$ with $i, j, k=1,2, \ldots$, $\left.\operatorname{dim} \mathcal{G}^{*}\right)$ is a Lie algebra.

Proposition: [41] From a 3-Lie algebra $\mathcal{A}_{\mathcal{G}}$ one constructs a 3-Lie bialgebra $\left(\mathcal{A}_{\mathcal{G}}, \mathcal{A}_{\mathcal{G}}^{*}\right)$ if and only if $\left(\mathcal{G}, \mathcal{G}^{*}\right)$ is a Lie bialgebra. The proof can be found in [41].

\section{BLG model on Manin of 3-Lie algebras (M2 $\leftrightarrow \mathrm{D} 2)$}

In the previous section we have considered a special case of the Manin triple $\left(\mathcal{D}, \mathcal{A}_{\mathcal{G}}, \mathcal{A}_{\mathcal{G}^{*}}\right)$ and have noted that there is a correspondence between the 3-Lie bialgebra $\left(\mathcal{A}_{\mathcal{G}}, \mathcal{A}_{\mathcal{G}^{*}}\right)$ and the Lie bialgebra $\left(\mathcal{G}, \mathcal{G}^{*}\right) .{ }^{5}$ Now we want to apply this 3 -Lie algebra $\mathcal{D}$ in the BLG model. We obtained in the previous section

$F_{C}^{-A B}=0, F_{C}^{\tilde{\sim} A B}=0$,

then

$F_{+}^{A B C}=0, F_{\tilde{+}}^{A B C}=0$.

\footnotetext{
${ }^{3}$ Note that this example of the 3-Lie bialgebra was considered in [41] for the first time. Here for self-containment of the paper we address it as an example.

${ }^{4}$ Note that the indices of $f_{k}^{i j}$ are lowered and raised by the ad-invariant metric $g^{i j}$ of the Lie algebra $\mathcal{G}$.

${ }^{5}$ In general the vector space $\mathcal{D}$ in the triple $\left(\mathcal{D}, \mathcal{A}_{\mathcal{G}}, \mathcal{A}_{\mathcal{G}^{*}}\right)$ is not 3-Lie algebra and also there is no correspondence between the Manin triple $\left(\mathcal{D}, \mathcal{A}_{\mathcal{G}}, \mathcal{A}_{\mathcal{G}^{*}}\right)$ and the Lie bialgebra $\left(\mathcal{G}, \mathcal{G}^{*}\right)$, but for the above special example $\mathcal{D}$ is a 3 -Lie algebra and for this case there is a correspondence.
} 
Note that for the Manin triple $\mathcal{D}$ we apply the symbol $F_{D}^{A B C}$ for the structure constant of the Manin triple as a $(4+2 \operatorname{dim} \mathcal{G})^{6}$ dimensional 3-Lie algebra, i.e. we have $T^{A}$ as a basis for the Manin triple with $A=+, T^{+}=T^{+}$; $A=-, T^{-}=T^{-} ; A=i, T^{i}=T^{i} ; A=i+(2+$ $\operatorname{dim} \mathcal{G}), T^{i+(2+\operatorname{dim} \mathcal{G})}=T^{\tilde{i}} ; A=(-)+(2+\operatorname{dim} \mathcal{G})$, $T^{(-)+(2+\operatorname{dim} \mathcal{G})}=T^{\tilde{-}}$ and $A=(+)+(2+\operatorname{dim} \mathcal{G})$, $T^{(+)+(2+\operatorname{dim} \mathcal{G})}=T^{\tilde{+}}$, together with the following commutation relations:

$$
\begin{aligned}
{\left[T^{-}, T^{A}, T^{B}\right] } & =0,\left[T^{+}, T^{i}, T^{j}\right]=f_{k}^{i j} T^{k}, \\
{\left[T^{+}, T^{i}, T^{\tilde{j}}\right] } & =f_{j}^{i k} T^{\tilde{k}},\left[T^{i}, T^{j}, T^{k}\right]=f^{i j k} T^{-}, \\
{\left[T^{\tilde{-}}, T^{A}, T^{B}\right] } & =0,\left[T^{\tilde{+}}, T^{\tilde{i}}, T^{\tilde{j}}\right]=\tilde{f}_{i j}^{k} T_{\tilde{k}}, \\
{\left[T^{\tilde{+}}, T^{\tilde{i}}, T^{j}\right] } & =\tilde{f}_{i k}^{j} T^{k},\left[T^{\tilde{i}}, T^{\tilde{j}}, T^{\tilde{k}}\right]=\tilde{f}_{i j k} T_{\tilde{\sim}}, \\
{\left[T^{\tilde{k}}, T^{i}, T^{j}\right] } & =f_{k}^{j i} T^{\tilde{-}},\left[T^{\tilde{+}}, T^{j}, T^{k}\right]=f^{i j k} T^{\tilde{i}}, \\
{\left[T^{+}, T^{\tilde{j}}, T^{\tilde{k}}\right] } & =\tilde{f}_{i j k} T^{i},\left[T^{k}, T^{\tilde{i}}, T^{\tilde{j}}\right]=-\tilde{f}_{i j}^{k} T^{-} .
\end{aligned}
$$

Now we write the equations of motion for the BLG model (10)-(12) by considering the 3-Lie algebra $\mathcal{D}$ of the Manin triple related to this special 3-Lie bialgebra as follows:

$$
\begin{aligned}
& \Gamma^{\mu} D_{\mu} \Psi_{A}+\frac{1}{2} \Gamma_{I J} X_{C}^{I} X_{D}^{J} \Psi_{B} F_{A}^{C D B}=0, \\
& D^{2} X_{A}^{I}-\frac{i}{2} \tilde{\Psi}_{C} \Gamma_{J}^{I} X_{D}^{J} \Psi_{B} F_{A}^{C D B} \\
& \quad+\frac{1}{2} F_{A}^{B C D} F_{D}^{E F G} X_{B}^{J} X_{C}^{K} X_{E}^{I} X_{F}^{J} X_{G}^{K}=0, \\
& \left(\hat{F}_{\mu \nu}\right)_{A}^{B}+\epsilon_{\mu \nu \lambda}\left(X_{C}^{J} D^{\lambda} X_{D}^{J}+\frac{i}{2} \bar{\Psi}_{C} \Gamma^{\lambda} \Psi_{D}\right) F_{A}^{C D B}=0,
\end{aligned}
$$

such that, if we take $A=+, \tilde{+}$ in (34) and (35), then we obtain the following relations:

$$
\begin{array}{ll}
\partial^{2} X_{+}^{I}=0, & \Gamma^{\mu} \partial_{\mu} \Psi_{+}=0, \\
\partial^{2} X_{\tilde{+}}^{I}=0, & \Gamma^{\mu} \partial_{\mu} \Psi_{\tilde{+}}=0,
\end{array}
$$

this means that $\left(X_{+}^{I}, X_{\tilde{\widetilde{\tau}}}^{I}\right)$ and $\left(\Psi_{+}, \Psi_{\tilde{+}}\right)$ can be set to a constant such as the Yang-Mills coupling [17] as applied to the whole theory and zero, respectively. This constant must conserve the SUSY transformations (4) for the Manin triple. Then, from the relations

$\delta X_{+}^{I}=i \bar{\epsilon} \Gamma^{I} \Psi_{+}$,

$\delta \Psi_{+}=\partial_{\mu} X_{+}^{I} \Gamma^{\mu} \Gamma^{I} \epsilon$,

one can show that the SUSY transformations do not change if we assign a vacuum expectation value (VEV) to one of the fields. Then the Lagrangian terms become as follows: by considering the relations

\footnotetext{
${ }^{6}$ We assume $\mathcal{G}$ and $\mathcal{G}^{*}$ have the same dimension i.e. $2+\operatorname{dim} \mathcal{G} ; 2$ is used for + and - .
}

$$
\begin{aligned}
D_{\mu} X_{-}^{(I)}= & \partial_{\mu} X_{-}^{(I)}+f^{i j k} A_{\mu j k} X_{i}^{(I)} \\
& +2 \tilde{f}_{j i}^{k} A_{\mu \tilde{j} k} X_{\tilde{i}}^{(I)}+\tilde{f}_{k j}^{i} A_{\mu \tilde{j} \tilde{k}} X_{i}^{(I)}, \\
D_{\mu} X_{\tilde{\sim}}^{(I)}= & \partial_{\mu} X_{\tilde{\sim}}^{(I)}+\tilde{f}_{i j k} A_{\mu \tilde{j} \tilde{k}} X_{\tilde{i}}^{(I)} \\
& +f_{i}^{j k} A_{\mu j k} X_{\tilde{i}}^{(I)}+2 f_{k}^{i j} A_{\mu j \tilde{k}} X_{i}^{(I)}, \\
D_{\mu} X_{i}^{(I)}= & \partial_{\mu} X_{i}^{(I)}+f_{i}^{j k} A_{\mu j k} X_{+}^{(I)}+\tilde{f}_{i j k} A_{\mu \tilde{j} \tilde{k}} X_{+}^{(I)} \\
& +2 f_{i}^{j k} A_{\mu k} X_{j}^{(I)}-2 \tilde{f}_{i j k} A_{\mu \tilde{k}} X_{\tilde{j}}^{(I)} \\
& +2 \tilde{f}_{j i}^{k} A_{\mu \tilde{j} k} X_{\tilde{+}}^{(I)}+2 f_{i j}^{k} A_{\mu k} X_{\tilde{j}}^{(I)}+2 \tilde{f}_{k i}^{j} A_{\mu \tilde{k}} X_{j}^{(I)}, \\
D_{\mu} X_{\tilde{i}}^{(I)}= & \partial_{\mu} X_{\tilde{i}}^{(I)}+f^{i j k} A_{\mu j k} X_{+}^{(I)}+\tilde{f}_{j k}^{i} A_{\mu \tilde{j} \tilde{k}} X_{\tilde{+}}^{(I)} \\
& -2 f^{i j k} A_{\mu k} X_{j}^{(I)}-\tilde{f}_{j k}^{i} A_{\mu \tilde{k}} X_{\tilde{j}}^{(I)} \\
& +2 f_{j}^{k i} A_{\mu \tilde{j} k} X_{+}^{(I)}-f_{j}^{k i} A_{\mu k} X_{\tilde{j}}^{(I)}-2 f_{k}^{i j} A_{\mu \tilde{k}} X_{j}^{(I)},
\end{aligned}
$$

we will have

$$
\begin{aligned}
D_{\mu} X_{A}^{(I)} D^{\mu} X^{A(I)}= & \partial_{\mu} X_{-}^{(I)} \partial^{\mu} X^{-(I)}+\partial_{\mu} X_{\tilde{-}}^{(I)} \partial^{\mu} X^{\tilde{-}(I)} \\
& +\partial_{\mu} X_{+}^{(I)} \partial^{\mu} X^{+(I)}+\partial_{\mu} X_{\tilde{+}}^{(I)} \partial^{\mu} X^{\tilde{+}(I)} \\
& +D_{\mu} X_{i}^{(I)} D^{\mu} X^{i(I)}+D_{\mu} X_{\tilde{i}}^{(I)} D^{\mu} X^{\tilde{i}(I)}
\end{aligned}
$$

where

$$
\begin{aligned}
D_{\mu} & X_{i}^{(I)} D^{\mu} X^{i(I)} \\
= & \partial_{\mu} X_{i}^{(I)} \partial^{\mu} X^{i(I)}+\left[f_{i}^{j k} A_{\mu j k} X_{+}^{(I)}\right. \\
& +2 \tilde{f}_{j i}^{k} A_{\mu \tilde{j k}} X_{\tilde{+}}^{(I)}+\tilde{f}_{i j k} A_{\mu \tilde{j} \tilde{k}} X_{+}^{(I)}-2 f_{i}^{j k} A_{\mu k} X_{j}^{(I)} \\
& -2 \tilde{f}_{i j k} A_{\mu \tilde{k}} X_{\tilde{j}}^{(I)}+2 f_{i j}^{k} A_{\mu k} X_{\tilde{j}}^{(I)} \\
& \left.+2 \tilde{f}_{k i}^{j} A_{\mu \tilde{k}} X_{j}^{(I)}\right]\left[f^{i j k} A_{\mu j k} X^{-(I)}-\tilde{f}_{j k}^{i} A_{\mu j \tilde{k}} X^{-(I)}\right. \\
& -2 f_{j}^{i k} A_{\mu k} X^{j(I)}+\tilde{f}_{j k}^{i} A \mu \tilde{k} X^{j(I)}+f_{k}^{i j} A_{\mu \tilde{k}} X^{\tilde{j}(I)} \\
& \left.-f^{i k j} A_{\mu k} X^{\tilde{j}(I)}-2 f_{k}^{i j} A_{\mu j \tilde{k}} X^{\tilde{-}(I)}\right]
\end{aligned}
$$

and

$$
\begin{aligned}
D_{\mu} & X_{\tilde{i}}^{(I)} D^{\mu} X^{\tilde{i}(I)} \\
= & \partial_{\mu} X_{\tilde{i}}^{(I)} \partial^{\mu} X^{\tilde{i}(I)}+\left[f^{i j k} A_{\mu j k} X_{+}^{(I)}\right. \\
& +\tilde{f}_{j k}^{i} A_{\mu \tilde{j} \tilde{k}} X_{\tilde{+}}^{(I)}+2 f^{k i} j A_{\mu \tilde{j} k} X_{+}^{(I)}-2 f^{i j k} A_{\mu k} X_{j}^{(I)} \\
& \left.-\tilde{f}_{j k}^{i} A_{\mu \tilde{k}} X_{\tilde{j}}^{(I)}-f_{j}^{k i} A_{\mu k} X_{\tilde{j}}^{(I)}-2 f_{k}^{i j} A_{\mu \tilde{k}} X_{j}^{(I)}\right] \\
& \times\left[2 \tilde{f}_{i k}^{j} A_{\mu j \tilde{k}} X^{-(I)}-2 f_{i}^{j k} A_{\mu j k} X^{\tilde{-}(I)}\right. \\
& -\tilde{f}_{i j k} A_{\mu \tilde{j} \tilde{k}} X^{\tilde{-}(I)}-2 f_{i k j} A_{\mu \tilde{k}} X^{j(I)} \\
& \left.-2 \tilde{f}_{j i}^{k} A_{\mu k} X^{j(I)}+2 f_{i}^{j k} A_{\mu k} X^{\tilde{j}(I)}+2 \tilde{f}_{i k}^{j} A_{\mu \tilde{k}} X^{\tilde{j}(I)}\right] .
\end{aligned}
$$


Furthermore, the first term of the CS term in the BLG action (14) turns into the following forms:

$$
\begin{aligned}
& \frac{1}{2} \epsilon^{\mu \nu \lambda} 7 F^{A B C D} A_{\mu A B} \partial_{\nu} A_{\lambda C D}=2 \epsilon^{\mu \nu \lambda} F^{B C D} \\
& \times A_{\mu B C} \partial_{\nu} A_{\lambda D}+2 \epsilon^{\mu \nu \lambda} F^{\mathcal{B C D}} A_{\mu \mathcal{B C}} \partial_{\nu} A_{\lambda \mathcal{D}}
\end{aligned}
$$

where

$$
\begin{aligned}
\epsilon^{\mu \nu \lambda} F^{B C D} A_{\mu B} \partial_{\nu} A_{\lambda C D}=\frac{1}{3} \epsilon^{\mu \nu \lambda} f_{i}^{j k} A_{\mu}^{i} \partial_{\nu} A_{\lambda j k} \\
+\frac{2}{3} \epsilon^{\mu \nu \lambda} f_{k}^{i j} A_{\mu i} \partial_{\nu} A_{\lambda j}^{k}+\frac{2}{3} \epsilon^{\mu \nu \lambda} f_{j}^{k i} A_{\mu i} \partial_{\nu} A_{\lambda \tilde{j}}^{\tilde{k}} \\
+\frac{2}{3} \epsilon^{\mu \nu \lambda} f_{k}^{i j} A_{\mu}^{\tilde{i}} \partial_{\nu} A_{\lambda j \tilde{k}}+\frac{2}{3} \epsilon^{\mu \nu \lambda} f_{i}^{j k} A_{\mu}^{\tilde{i}} \partial_{\nu} A_{\lambda j}^{\tilde{k}} \\
+\frac{1}{3} \epsilon^{\mu \nu \lambda} \tilde{f}_{j k}^{i} A_{\mu}^{\tilde{i}} \partial_{\nu} A_{\lambda \tilde{j} \tilde{k}}+\frac{2}{3} \epsilon^{\mu \nu \lambda} \tilde{f}_{i k}^{j} A_{\mu}^{i} \partial_{\nu} A_{\lambda j \tilde{k}} \\
+\frac{2}{3} \epsilon^{\mu \nu \lambda} \tilde{f}_{j k}^{i} A_{\mu}^{i} \partial_{\nu} A_{\lambda \tilde{k}}^{j}+\frac{2}{3} \epsilon^{\mu \nu \lambda} \tilde{f}_{k \tilde{i}}^{j} A_{\mu \tilde{i}} \partial_{\nu} A_{\lambda j}^{k} \\
\quad+\frac{2}{3} \epsilon^{\mu \nu \lambda} \tilde{f}_{i j}^{k} A_{\mu \tilde{i}} \partial_{\nu} A_{\lambda \tilde{k}}^{\tilde{k}}
\end{aligned}
$$

and

$$
\begin{aligned}
\epsilon^{\mu \nu \lambda} F^{\mathcal{B C D}} A_{\mu \mathcal{B}} \partial_{\nu} A_{\lambda \mathcal{C D}}= & \frac{2}{3} \epsilon^{\mu \nu \lambda} f^{i j k} A_{\mu i} \partial_{\nu} A_{\lambda j}^{\tilde{k}} \\
& +\frac{1}{3} \epsilon^{\mu \nu \lambda} f_{i}^{j k i} A_{\mu}^{\tilde{i}} \partial_{\nu} A_{\lambda j k} \\
& +\frac{1}{3} \epsilon^{\mu \nu \lambda} \tilde{f}_{i j k}^{i} A_{\mu}^{i} \partial_{\nu} A_{\lambda \tilde{j} \tilde{k}} \\
& +\frac{2}{3} \epsilon^{\mu \nu \lambda} \tilde{f}_{j i k}^{i} A_{\mu \tilde{i}} \partial_{\nu} A_{\lambda \tilde{k}}^{j}
\end{aligned}
$$

and the second term of the CS term in the BLG action

$$
\begin{aligned}
\frac{1}{3} \epsilon^{\mu \nu \lambda} F_{G}^{A E F} F^{B C D G} A_{\mu A B} A_{\nu C D} A_{\lambda E F} \\
=-2 \epsilon^{\mu \nu \lambda} F^{A B C} F_{A}^{E F} A_{\mu E F} A_{\nu B} A_{\lambda C} \\
-2 \epsilon^{\mu \nu \lambda} F^{\mathcal{A B C}} F_{\mathcal{A}}^{\mathcal{E F}} A_{\mu \mathcal{E} \mathcal{F}} A_{\nu \mathcal{B}} A_{\lambda \mathcal{C}}
\end{aligned}
$$

where

$$
\begin{aligned}
\epsilon^{\mu \nu \lambda} & F^{A B C} F_{A}^{E F} A_{\mu E F} A_{\nu B} A_{\lambda C} \\
= & \frac{1}{2} \epsilon^{\mu \nu \lambda} f_{i}^{j k} f_{m}^{i l} A_{\mu j k} A_{\nu l} A_{\lambda}^{m}+\frac{1}{2} \epsilon^{\mu \nu \lambda} f_{k}^{i j} f_{i}^{l m} A_{\mu j}^{k} A_{\nu l} A_{\lambda m} \\
& +\epsilon^{\mu \nu \lambda} f_{k}^{i j} \tilde{f}_{m i}^{l} A_{\mu j \tilde{k}} A_{\nu l} A_{\lambda}^{m}+\frac{1}{2} \epsilon^{\mu \nu \lambda} f_{i}^{j k} \tilde{f}_{l m}^{i} A_{\mu j k} A_{\nu \tilde{l}} A_{\lambda}^{m} \\
& +\epsilon^{\mu \nu \lambda} f_{k}^{i j} \tilde{f}_{i l}^{m} A_{\mu j}^{k} A_{\nu \tilde{l}} A_{\lambda m}+\epsilon^{\mu \nu \lambda} f_{k}^{i j} f_{i}^{l m} A_{\mu j \tilde{k}} A_{\nu}^{\tilde{l}} A_{\lambda m} \\
& +\frac{1}{2} \epsilon^{\mu \nu \lambda} f_{i}^{j k} f_{l}^{m i} A_{\mu j}^{\tilde{k}} A_{\nu \tilde{l}} A_{\lambda m}+\epsilon^{\mu \nu \lambda} f_{l}^{m i} \tilde{f}_{k i}^{j} A_{\mu j \tilde{k}} A_{\nu}^{l} A_{\lambda m} \\
& +\epsilon^{\mu \nu \lambda} f_{i}^{l m} \tilde{f}_{k j}^{i} A_{\mu \tilde{k}}^{j} A_{\nu l} A_{\lambda m}+\frac{1}{2} \epsilon^{\mu \nu \lambda} \tilde{f}_{j k}^{i} \tilde{f}_{m i}^{l} A_{\mu \tilde{j} \tilde{k}} A_{\nu l} A_{\lambda}^{m} \\
& +\epsilon^{\mu \nu \lambda} \tilde{f}_{k i}^{j} \tilde{f}_{l m}^{i} A_{\mu \tilde{j} \tilde{k}} A_{\nu \tilde{l}} A_{\lambda}^{m}+\epsilon^{\mu \nu \lambda} \tilde{f}_{k j}^{i} \tilde{f}_{i l}^{m} A_{\mu \tilde{k}}^{j} A_{\nu \tilde{l}} A_{\lambda m} \\
& +\frac{1}{2} \epsilon^{\mu \nu \lambda} f_{l}^{i m} \tilde{f}_{i j}^{k} A_{\mu \tilde{j}}^{\tilde{k}} A_{\nu \tilde{l}} A_{\lambda m}+\frac{1}{4} \epsilon^{\mu \nu \lambda} f_{i}^{l m} \tilde{f}_{j k}^{i} A_{\mu \tilde{j} \tilde{k}} A_{\nu l} A_{\lambda}^{\tilde{m}} \\
& +\epsilon^{\mu \nu \lambda} f_{l}^{m i} \tilde{f}_{k i}^{j} A_{\mu j \tilde{k}} A_{\nu \tilde{l}} A_{\lambda}^{\tilde{m}}+\frac{1}{2} \epsilon^{\mu \nu \lambda} \tilde{f}_{j k}^{i} \tilde{f}_{m i}^{l} A_{\mu \tilde{j} \tilde{k}} A_{\nu}^{\tilde{l}} A_{\lambda \tilde{m}}
\end{aligned}
$$

$$
\begin{aligned}
& +\frac{1}{2} \epsilon^{\mu \nu \lambda} \tilde{f}_{i j}^{k} \tilde{f}_{l m}^{i} A_{\mu \tilde{j}}^{\tilde{k}} A_{\nu \tilde{l}} A_{\lambda \tilde{m}}+\frac{1}{2} \epsilon^{\mu \nu \lambda} f_{i}^{j k} \tilde{f}_{l i}^{m} A_{\mu j}^{\tilde{k}} A_{\nu l} A_{\lambda m} \\
& +\frac{1}{2} \epsilon^{\mu \nu \lambda} f_{i}^{j k} f_{l}^{m i} A_{\mu j k} A_{\nu \tilde{l}} A_{\lambda}^{\tilde{m}}+\epsilon^{\mu \nu \lambda} f_{k}^{i j} \tilde{f}_{i l}^{m} A_{\mu j \tilde{k}} A_{\nu \tilde{l}} A_{\lambda}^{\tilde{m}} \\
& +\frac{1}{2} \epsilon^{\mu \nu \lambda} f_{i}^{j k} \tilde{f}_{l m}^{i} A_{\mu j \tilde{k}} A_{\nu \tilde{l}} A_{\lambda \tilde{m}}
\end{aligned}
$$

and

$$
\begin{aligned}
& \epsilon^{\mu \nu \lambda} F^{\mathcal{A B C}} F_{\mathcal{A}}^{\mathcal{E F}} A_{\mu \mathcal{E F}} A_{\nu \mathcal{B}} A_{\lambda \mathcal{C}} \\
& =\frac{1}{3} \epsilon^{\mu \nu \lambda}\left(-\left[2 f_{i}^{j k} f_{m}^{i l}+\frac{3}{2} f^{j k i} \tilde{f}_{m i}^{l}+3 f^{l j i} \tilde{f} m i^{k}\right] A_{\mu j k} A_{\nu l} A_{\lambda}^{m}\right. \\
& +\left[2 f_{i}^{k j} f_{m}^{l i}-3 f^{l k i} \tilde{f}_{m i}^{j}-2 f^{j i l} \tilde{f}_{m i}^{k}\right] A_{\mu \tilde{m}} A_{\nu k}^{\tilde{j}} A_{\lambda l} \\
& -\left[f_{i}^{j k} f_{l}^{i m}-2 f^{i j m} \tilde{f}_{i l}^{k}+\tilde{f}_{i l}^{m} f^{j k i}\right] A_{\mu}^{\tilde{m}} A_{v j k} A_{\lambda \tilde{l}} \\
& -2\left[2 f_{j}^{k i} \tilde{f}_{l i}^{m}-\frac{1}{2} f^{i k m} \tilde{f}_{i l j}+2 f_{l}^{i m} \tilde{f}_{j i}^{k}-\frac{1}{2} f_{i}^{m k} \tilde{f}_{l j}^{i}\right. \\
& \left.-\frac{1}{2} f_{l}^{k i} \tilde{f}_{i j}^{m}-\frac{1}{2} f^{k i m} \tilde{f}_{i l j}\right] A_{\mu}^{\tilde{m}} A_{\nu \tilde{j} k} A_{\lambda \tilde{l}}+3 f_{m}^{i l} \tilde{f}_{k i j} A_{\mu k} A_{\nu \tilde{j}} A_{\lambda l \tilde{m}} \\
& +\left[f^{j k i} \tilde{f}_{i m}^{l}+f^{l k i} \tilde{f}_{m i}^{j}+2 f_{i}^{j k} f_{m}^{l i}\right] A_{\mu k}^{m} A_{\nu}^{\tilde{j}} A_{\lambda l} \\
& -\left[\tilde{f}_{j k}^{i} \tilde{f}_{i l}^{m}+\tilde{f}_{m k}^{i} \tilde{f}_{i l m}\right] A_{\mu \tilde{j}} A_{\nu \tilde{j}}^{k} A_{\lambda \tilde{l}}+\tilde{f}_{j i k} f_{l}^{i m} A_{\mu m}^{\tilde{k}} A_{\nu}^{j} A_{\lambda l} \\
& -\left[2 \tilde{f}_{m l}^{i} f_{i}^{j k}+2 \tilde{f}_{i l}^{k} f_{m}^{i j}+\frac{1}{2} \tilde{f}_{m i l} f^{j k i}+2 \tilde{f}_{i l}^{k} f_{m}^{i j}+\tilde{f}_{m i}^{j} f_{l}^{k i}\right. \\
& \left.+\tilde{f}_{l m}^{i} f_{i}^{j k}\right] A_{\mu}^{m} A_{v j k} A_{\lambda \tilde{l}}+\tilde{f}_{i m}^{l} f_{k}^{j i} A_{\mu \tilde{m}}^{k} A_{\nu}^{\tilde{j}} A_{\lambda l} \\
& +\left[f^{l m i} f_{i}^{j k}-f^{j l i} f_{i}^{m k}-2 f^{i m k} f_{i}^{j l}+2 f^{i j k} f_{i}^{l m} \tilde{f}_{m i}^{l} f^{i j k}\right. \\
& \left.-\frac{1}{2} f^{l m i} f_{i}^{k j}\right] A_{\mu}^{\tilde{k}} A_{\nu j} A_{\lambda l m}+\tilde{f}_{i j k} f^{l i m} A_{\mu}^{\tilde{m}} A_{\nu \tilde{j} \tilde{k}} A_{\lambda m}^{\tilde{l}} \\
& -2\left[f_{j}^{k i} f_{i}^{l m}+f_{i}^{m k} f_{j}^{i l} A_{\mu \tilde{m}}^{\tilde{k}} A_{\nu j} A_{\lambda l}+\frac{1}{2} \tilde{f}_{j i}^{k} f^{m l i}\right. \\
& \left.+\frac{1}{2} \tilde{f}_{l i}^{m} f_{j}^{i k}-\tilde{f}_{j i}^{l} f^{m i k}\right] A_{\mu m} A_{v \tilde{j}}^{\tilde{k}} A_{\lambda l}+2 f_{j}^{k i} f_{i}^{l m} A_{\mu k m} A_{\nu \tilde{j}} A_{\lambda}^{\tilde{l}} \\
& -\left[f_{i}^{m l} \tilde{f}_{j k}^{i}-f_{k}^{m i} \tilde{f}_{j i}^{l}+2 f_{k}^{i l} \tilde{f}_{i j}^{m}\right] A_{\mu}^{\tilde{m}} A_{\nu \tilde{j} \tilde{k}} A_{\lambda l}+3\left[-\frac{1}{2} \tilde{f}_{j i k} \tilde{f}_{l m}^{i}\right. \\
& \left.+\frac{2}{3} \epsilon^{\mu \nu \lambda} \tilde{f}_{k l}^{i} \tilde{f}_{j i m}\right] A_{\mu \tilde{k}} A_{\nu}^{j} A_{\lambda \tilde{l} \tilde{m}}-\tilde{f}_{i k}^{j} f_{m}^{i l} A_{\mu}^{\tilde{k}} A_{\nu}^{\tilde{j}} A_{\lambda l \tilde{m}} \\
& +\left[\tilde{f}_{j k}^{i} \tilde{f}_{i m}^{l}+2 \tilde{f}_{j i}^{l} \tilde{f}_{k m}^{i}+2 \tilde{f}_{m i k} f_{j}^{i l}+\tilde{f}_{i j k} f_{m}^{l i}\right] A_{\mu}^{m} A_{v \tilde{j} \tilde{k}} A_{\lambda l} \\
& +\left[\tilde{f}_{m l}^{i} f_{i}^{j k}-\tilde{f}_{l i}^{k} f_{m}^{i j}-2 f_{l}^{i j} \tilde{f}_{i m}^{k} A-2 f^{i j k} \tilde{f}_{m l i}\right] A_{\mu k} A_{\nu j} A_{\lambda \tilde{l}}^{m} \\
& -\left[3 \tilde{f}_{i k}^{j} \tilde{f}_{m l}^{i}+\tilde{f}_{i m}^{j} \tilde{f}_{k l i}+2 \tilde{f}_{i l k} f_{m}^{j i}\right] A_{\mu \tilde{k} \tilde{m}} A_{\nu}^{\tilde{j}} A_{\lambda \tilde{l}} \\
& -2\left[2 \tilde{f}_{i j}^{k} \tilde{f}_{m l}^{i}-f_{m}^{k i} \tilde{f}_{i l j} A_{\mu \tilde{m}}-f_{j}^{k i} \tilde{f}_{i l m} A_{\mu \tilde{m}}^{\tilde{k}}\right. \\
& \left.-\frac{1}{2} \tilde{f}_{m i}^{l} f_{l}^{i k}\right] A_{\mu \tilde{m}}^{\tilde{k}} A_{\nu \tilde{j}} A_{\lambda \tilde{l}} \\
& -\left[2 \tilde{f}_{i j}^{k} f_{l}^{i m}-3 f_{j}^{k i} \tilde{f}_{l i}^{m}+2 \tilde{f}_{i l}^{k} f_{j}^{i m}+\tilde{f}_{l k}^{i} f_{i}^{m j}\right] A_{\mu m} A_{\nu \tilde{j}}^{\tilde{k}} A_{\lambda \tilde{l}}\left[\tilde{f}_{i m}^{l} \tilde{f}_{j k}^{i}\right. \\
& \left.+2 f_{j}^{i l} \tilde{f}_{k i m}+3 \tilde{f}_{m j}^{i} \tilde{f}_{i k}^{l}+2 f_{l}^{j i} \tilde{f}_{k m}^{i}\right] A_{\mu \tilde{m}} A_{\nu \tilde{j}}^{k} A_{\lambda l} \\
& -\left[2 f_{k}^{i j} \tilde{f}_{i l}^{m}-2 f_{i}^{j m} \tilde{f}_{k l}^{i}-2 f_{k}^{i m} \tilde{f}_{i l}^{j}+f_{k}^{i m} \tilde{f}_{i j l}-f_{l}^{k j} f_{i}^{j m}\right. \\
& +2 f_{l}^{i j} \tilde{f}_{k i}^{m}-f^{m i j} \tilde{f}_{k i l}+\tilde{f}_{j k}^{i} \tilde{f}_{m i}^{l}+f^{j m i} \tilde{f}_{k i l} \\
& \left.+2 f_{l}^{m i} \tilde{f}_{i k}^{j}\right] A_{\mu}^{k} A_{v j} A_{\lambda \tilde{l} m}-\left[2 f_{j}^{k i} f_{i}^{m l}+\tilde{f}_{j i}^{k} f^{i l m}+2 \tilde{f}_{j i}^{l} f^{i m k}\right. \\
& \left.-3 f^{k i l} \tilde{f}_{i j}^{m}\right] A_{\mu}^{\tilde{m}} A_{\nu \tilde{j} k} A_{\lambda l}+\tilde{f}_{k i}^{j} f^{l m i} A_{\mu m} A_{v j}^{k} A_{\lambda l} \\
& -\left[4 \tilde{f}_{i j}^{k} \tilde{f}_{m l}^{i}-f_{l}^{i k} \tilde{f}_{i m j}+f_{m}^{k i} \tilde{f}_{l i j} A_{\mu k \tilde{m}}\right] A_{\mu \tilde{m}} A_{\nu \tilde{j} k} A_{\lambda}^{l} \\
& +2\left[\tilde{f}_{j k}^{i} \tilde{f}_{l i}^{m}+\tilde{f}_{i j}^{m} \tilde{f}_{k l}^{i}\right] A_{\mu \tilde{k} m} A_{\nu \tilde{j}} A_{\lambda}^{l}+\left[2 \tilde{f}_{j k}^{i} f_{i}^{m l}+f_{j}^{i m} \tilde{f}_{k i}^{l}\right.
\end{aligned}
$$




$$
\begin{aligned}
& \left.-2 \tilde{f}_{j k i} \tilde{f}_{l i m}+f_{k}^{l i} \tilde{f}_{i j}^{m}-f_{k}^{i m} \tilde{f}_{i j}^{l}\right] A_{\mu \tilde{k}} A_{\nu \tilde{j}} A_{\lambda l}^{\tilde{m}} \\
& \left.-\left[2 f_{m}^{l i} \tilde{f}_{i j}^{k}+\tilde{f}_{i j l} f_{m}^{k i}-f_{m}^{i k} \tilde{f}_{i j}^{l}-f_{j}^{i k} \tilde{f}_{i m}^{l}-f_{j}^{l i} \tilde{f}_{m i}^{k}\right] A_{\mu k} A_{\nu \tilde{j}} A_{\lambda l}^{m}\right),
\end{aligned}
$$

where $F^{A B C}$ is the structure constant of the Manin triple of the Lie bialgebra $\left(\mathcal{D}, \mathcal{G}, \mathcal{G}^{*}\right){ }^{7}$ Note that the indices $A$ and $\mathcal{A}$ can be $i$ and $\tilde{i}$. In the above relations we have used the notations $A_{\mu+B}=A_{\mu \tilde{+} B}=A_{\mu B}, A_{\mu+\tilde{B}}=A_{\mu \tilde{+} \tilde{B}}=A_{\mu \tilde{B}}$, $F_{C}^{A B} A_{\mu A B} \equiv C_{\mu C}$ and $F_{\mathcal{C}}^{\mathcal{A B}} A_{\mu \mathcal{A B}} \equiv \tilde{C}_{\mu \mathcal{C}}$ that $\mathcal{A}=i, \tilde{i}$ and if they cannot come only from $\mathcal{G}$ or $\mathcal{G}^{*}$, then the sum of (47) and (50) will have the following form:

$$
\begin{gathered}
\frac{1}{2} \epsilon^{\mu \nu \lambda}\left\{C_{\mu B}\left(\partial_{\nu} A_{\lambda}^{B}-\partial_{\lambda} A_{\nu}^{B}-\left[A_{\nu}, A_{\lambda}\right]_{B}\right)\right. \\
\left.+\tilde{C}_{\mu}^{\mathcal{B}}\left(\partial_{\nu} A_{\lambda \mathcal{B}}-\partial_{\lambda} A_{\nu \mathcal{B}}-\left[A_{\nu}, A_{\lambda}\right]_{\mathcal{B}}\right)\right\} .
\end{gathered}
$$

In this way the general form of the BLG Lagrangian on the special 3-Lie algebra (Manin triple) (33) is as follows: ${ }^{8}$

$$
\begin{aligned}
L= & \frac{1}{2} D_{\mu} X^{A(I)} D^{\mu} X_{A}^{(I)}-2 g_{Y M}^{2} C_{\mu}^{B} C_{B}^{\mu} \\
& -2 g_{Y M} \tilde{C}_{\mu \mathcal{B}} D^{\mu} X^{(8) \mathcal{B}}+2 \epsilon^{\mu \nu \lambda} C_{\mu A} B_{\nu \lambda}^{A} \\
& +2 \epsilon^{\mu \nu \lambda} \tilde{C}_{\mu}^{\mathcal{B}} F_{\nu \lambda \mathcal{B}}+E, \\
E= & g_{Y M} C^{\mu i} \partial_{\mu} X_{i}^{(I)}+g_{Y M} C_{\mu i} \partial^{\mu} X^{i(I)} \\
& +C_{\mu i} \tilde{C}^{\mu i}-2 g_{Y M} f^{i k j} A_{\mu k} X^{j(I)} C_{\mu i}+g_{Y M} \tilde{f}_{j k}^{i} A_{\mu \tilde{k}} C_{\mu i} \\
& +g_{Y M} f_{k}^{i j} A_{\mu \tilde{k}} X^{\tilde{j}(I)} C_{\mu i}+g_{Y M} f^{i k j} A_{\mu k} X^{\tilde{j}(I)} C_{\mu i}+\cdots,
\end{aligned}
$$

where

$$
\begin{aligned}
B_{v \lambda A} & =\partial_{\nu} A_{\lambda A}-\partial_{\lambda} A_{\nu A}-\left[A_{\nu}, A_{\lambda}\right]_{A}, \\
F_{\nu \lambda}^{\mathcal{A}} & =\partial_{\nu} A_{\lambda}^{\mathcal{A}}-\partial_{\lambda} A_{\nu}^{\mathcal{A}}-\left[A_{\nu}, A_{\lambda}\right]^{\mathcal{A}} .
\end{aligned}
$$

Now, by integration of $C_{\mu k}$ and $\tilde{C}_{\mu}^{k}$,

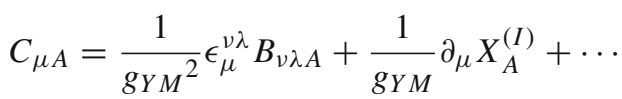

\footnotetext{
${ }^{7}$ Note that a 3 -Lie bialgebra is not a direct sum of $\mathcal{A}$ and $\mathcal{A}^{*}$; if so, we could not have the relation $\left[T^{+}, T^{i}, T^{\tilde{j}}\right]=f^{i k} j T^{\tilde{k}}$ and $\left[T^{\tilde{+}}, T^{\tilde{i}}, T^{j}\right]=\tilde{f}_{i k}^{j} T^{k}$ and the proposition in the previous section will fail for this case. Now, as we investigate this case i.e. the direct sum for our model, then Eq. (48) turns into the following form:

$\epsilon^{\mu \nu \lambda} F^{B C D} A_{\mu B} \partial_{\nu} A_{\lambda C D}=f_{k}^{i j} A_{\mu i} \partial_{\nu} A_{\lambda j}^{k}+\tilde{f}_{i j}^{k} A_{\mu \tilde{i}} \partial_{\nu} A_{\lambda \tilde{k} \tilde{k}}$

and Eq. (51) as follows:

$$
\begin{aligned}
\epsilon^{\mu \nu \lambda} F^{A B C} F_{A}^{E F} A_{\mu E F} A_{\nu B} A_{\lambda C}= & \epsilon^{\mu \nu \lambda} f_{i}^{j k} f_{m}^{i l} A_{\mu l}^{m} A_{\nu j} A_{\lambda k} \\
& +\epsilon^{\mu \nu \lambda} \tilde{f}_{k i}^{j} \tilde{f}_{m l}^{i} A_{\mu \tilde{m}}^{l} A_{\nu j} A_{\lambda \tilde{k}} ;
\end{aligned}
$$

the result is the same as in Ref. [18] with one difference: we will have two Yang-Mills actions, one for $\mathcal{A}$ and the other for $\mathcal{A}^{*}$.

8 Note that in this Lagrangian the $E$ term (also “+ $+\ldots$ " terms) according to Eqs. (44)-(46) cannot contribute in Yang-Mills and DBI actions.
}

and

$\tilde{C}_{\mu \mathcal{A}}=\frac{1}{g_{Y M}{ }^{2}} \epsilon_{\mu}^{\nu \lambda} F_{\nu \lambda \mathcal{A}}+\frac{1}{g_{Y M}} \partial_{\mu} X_{\mathcal{A}}^{(I)}+\cdots$

then by insertion in the Lagrangian we will obtain the following equation:

$L=\frac{1}{2} F_{\nu \lambda \mathcal{A}} F^{\nu \lambda \mathcal{A}}+\frac{1}{2} B_{\nu \lambda A} B^{\nu \lambda A}+\frac{1}{2} D_{\mu} X^{A(I)} D^{\mu} X_{A}^{(I)}+\cdots$,

where $F_{v \lambda \mathcal{A}}$ is the field strength of Yang-Mills (with the gauge field $A_{\lambda A}$ ) and $B_{\nu \lambda A}$ is related to the B-field of a string. As is well known, the dynamic of D-branes expressed by the DBI and the Yang-Mills action can be obtained by expanding the DBI action [42-44]. Furthermore, the relation between the DBI action and the sigma model has been investigated by Leigh in Ref. [45], so the relation between the DBI action and the WZW models that are sigma models on Lie groups may exist. In this way, our claim might be true that expanding the DBI action leads to a term that corresponds to a B-field of a WZW model. We must consider that fields are 3-Lie algebraic valued in this WZW model of which we will obtain the form in the next subsection. Therefore, we will have the WZW model from the BLG model, which has been constructed on a Manin triple; otherwise we will have the Yang-Mills model from it [18]. In this way, we provide a method to obtain the D2 case from the M2 case, and vice versa. Note that this method for obtaining D2 from M2 is different from the method of Ref. $[15,16]$. In the reference mentioned one cannot obtain M2 from D2 but in our method this is possible. The BLG model is maximally supersymmetric $(N=8)$ in $2+1$ dimensions, with the correlating DBI action being $N=(4,4)$ supersymmetric or a string being $N=(4,4)$ supersymmetric. We know that if the string propagates on a group manifold, one can replace the string action with the WZW action. Therefore, if we assume that our string model propagates on a Lie group, then we will have an $N=(4,4)$ supersymmetric WZW model in two dimensions. In the previous work we analyzed the algebraic structure of the $N=(4,4)$ supersymmetric WZW model and showed that this model has a Lie bialgebra structure with one 2-cocycle [31]. Therefore, the Lie algebra $\mathcal{G}$ in (29) must have a Lie bialgebraic structure with 2-cocycles. In this way by starting with an $N=(4,4) \mathrm{WZW}$ model (D2-model) with the Lie algebra $\mathcal{G}$ (where it is a Lie bialgebra with one 2-cocycle) one can obtain a BLG model (M2-model) by a 3-Lie algebra with the commutation relation (33), which is obtained from $\mathcal{G}$ and its dual $\mathcal{G}^{*}$. Note that, contrary to the ordinary WZW model, here in this model the B-field has an algebraic index and therefore we have an $N=(4,4)$ like WZW model (the form of the B-field is shown in (58)). 


\subsection{WZW model with 3-Lie algebra valued fields}

We know that the WZW action has the following form [46]:

$$
\begin{aligned}
S_{\mathrm{WZW}}= & \int d^{3} x \epsilon^{\alpha \beta \gamma} L_{\mu}^{I} L_{\nu}^{J} L_{\lambda}^{K} \\
& \times \partial_{\alpha} X^{\mu} \partial_{\beta} X^{\nu} \partial_{\gamma} X^{\lambda} \operatorname{Tr}\left(\left[T_{I}, T_{J}\right], T_{K}\right) .
\end{aligned}
$$

Now by setting the algebraic index for the space-time coordinate $\left(X^{\mu}=X^{\mu A} T_{A}\right)$ one can write the WZW-like term in the following form:

$$
\begin{aligned}
& S_{\mathrm{WZW}-\text { like }}=\int d^{3} x \epsilon^{\alpha \beta \gamma} L_{\mu}^{L} L_{\nu}^{M} L_{\lambda}^{N} \\
& \quad \times \partial_{\alpha} X^{I \mu} \partial_{\beta} X^{J v} \partial_{\gamma} X^{K \lambda} \operatorname{Tr}\left(\left[T_{I} T_{L}, T_{J} T_{M}\right], T_{K} T_{N}\right),
\end{aligned}
$$

subsequently, we anticipate that the B-field of the WZW-like model has two algebraic indices as in the following form:

$$
\begin{aligned}
S_{\mathrm{WZW}-\text { like }}= & \int d^{2} x\left\{\frac{1}{6} \epsilon^{\beta \gamma} B_{v \mu} Q_{\partial_{\beta}} X^{J v} \partial_{\gamma} X^{I \mu} \operatorname{Tr}\left(T_{J} T_{I} T_{Q}\right)\right. \\
& +\frac{1}{6} \epsilon^{\alpha \gamma} B_{v \lambda}{ }^{Q} \partial_{\alpha} X^{J v} \partial_{\gamma} X^{K \lambda} \operatorname{Tr}\left(T_{J} T_{K} T_{Q}\right) \\
& \left.+\frac{1}{6} \epsilon^{\alpha \gamma} B_{\mu \lambda} Q_{\partial_{\alpha}} X^{I \mu} \partial_{\gamma} X^{K \lambda} \operatorname{Tr}\left(T_{I} T_{K} T_{Q}\right)\right\}+\cdots,
\end{aligned}
$$

where $B_{v \mu}{ }^{Q}=L_{v}{ }^{L} L_{\lambda}{ }^{N} f_{N L}{ }^{P} x^{J} f_{P J}{ }^{Q},\left.L_{\mu}^{L} X^{I \mu} T_{I} T_{L}\right|_{\text {boundary }}$ $=\left.x^{L} T_{L}\right|_{\text {boundary }}$ such that the $B_{v \mu} Q$ have the form of (58), and in this way the kinetic term for the WZW-like action has the following form:

$$
\int L_{\mu}^{L} L_{v}^{M} \partial X^{I \mu} \partial X^{J v} f_{L M}^{Q} \operatorname{Tr}\left(T_{Q} T_{I} T_{J}\right) .
$$

Therefore, we see that the second term of (62) is related to the WZW-like action, i.e., a $N=(4,4)$ string (D2-model) with the Lie algebra $\mathcal{G}$ as a Manin triple of Lie bialgebra with one 2 -cocycle. This means that if we have an $N=(4,4) \mathrm{WZW}$ model (D2-model) with Lie algebra $\mathcal{G}$ (as a Manin triple of a Lie bialgebra with one 2-cocycle) we will have a BLG model M2-model) related to 3-Lie algebra [42,43] (which is obtained from $\mathcal{G}$ and $\mathcal{G}^{*}$ ) and vice versa.

\section{Conclusions}

Using the concept of the 3-Lie bialgebra (recently defined in arXiv:1604.04475) we have constructed the BLG model on a Manin triple of a special 3-Lie bialgebra $\left(\mathcal{D}, \mathcal{A}, \mathcal{A}^{*}\right)$. Then, using the correspondence between the 3-Lie bialgebra $\left(\mathcal{A}, \mathcal{A}^{*}\right)$ and the Lie bialgebra $\left(\mathcal{G}, \mathcal{G}^{*}\right)$ we have shown that the BLG model can be reduced to an $N=(4,4)$ WZW model on a Lie algebra $\mathcal{G}$ such that the Lie algebra had one 2-cocycle.

In this way one can begin with a D2-model with the Lie algebra $\mathcal{G}$ and construct the M2-model over $\mathcal{D}$ and vice versa. One of the open problems is whether one can classify such
M2-models by using the classification of the Lie bialgebras $\mathcal{G}$.

Acknowledgements We would like to thank M. Akbari-Moghanjoughi for carefully reading the manuscript. This research was supported by a research fund No. 217D4310 of Azarbaijan Shahid Madani university.

Open Access This article is distributed under the terms of the Creative Commons Attribution 4.0 International License (http://creativecomm ons.org/licenses/by/4.0/), which permits unrestricted use, distribution, and reproduction in any medium, provided you give appropriate credit to the original author(s) and the source, provide a link to the Creative Commons license, and indicate if changes were made.

Funded by SCOAP 3 .

\section{Appendix}

\section{The adjoint representation of the 3 -Lie algebra and ad-invariant metric on $\mathcal{D}$}

Here we assume that $\mathcal{D}$ of the Manin triple $\left(\mathcal{D}, \mathcal{A}, \mathcal{A}^{*}\right)$ is a 3-Lie algebra.

A Manin triple $\left(D, \mathcal{A}, \mathcal{A}^{*}\right)$ which is in one-to-one correspondence with 3 -Lie bialgebras $\left(\mathcal{A}, \mathcal{A}^{*}\right)$ must have a nondegenerate ad-invariant inner product over the 3-Lie algebra $\mathcal{D}$. In order to calculate this we choose the basis of $\mathcal{D}=\mathcal{A} \oplus \mathcal{A}^{*}$ as $T^{A}\left(\left\{T^{-}, T^{+}, T^{i}, \tilde{T}_{-}, \tilde{T}_{+}, \tilde{T}_{i}\right\}\right)=$ $\left\{T^{-}, T^{+}, T^{i}, T^{\tilde{\mathcal{A}}}, T^{\tilde{+}}, T^{\tilde{i}}\right\}$ and use the commutation relation on $\mathcal{A}$ and $\mathcal{A}^{*}$ :

$$
\begin{aligned}
& {\left[T^{-}, T^{a}, T^{b}\right]=0,} \\
& {\left[T^{+}, T^{i}, T^{j}\right]=f_{k}^{i j} T^{k},} \\
& {\left[T^{i}, T^{j}, T^{k}\right]=f^{i j k} T^{-},} \\
& {\left[\tilde{T}_{-}, \tilde{T}_{a}, \tilde{T}_{b}\right]=0,} \\
& {\left[\tilde{T}_{+}, \tilde{T}_{i}, \tilde{T}_{j}\right]=\tilde{f}_{i j}^{k} \tilde{T}_{k},} \\
& {\left[\tilde{T}_{i}, \tilde{T}_{j}, \tilde{T}_{k}\right]=\tilde{f}_{i j k} T_{-} .}
\end{aligned}
$$

Now, the commutation relation for the 3-Lie algebras $\mathcal{D}$ has the following form:

$\left[T^{A}, T^{B}, T^{C}\right]=F_{D}^{A B C} T^{D}$,

which we use for the simplification:

$$
\left(\begin{array}{llll}
a & b & c & d \\
e & f & g & h \\
i & j & k & l \\
m & n & p & q
\end{array}\right),
$$

where $a, c, i, k$ are $2 \times 2$ matrices, $b, d, j, l$ are $2 \times n$ matrices, $e, g, m, p$ are $n \times 2$ matrices and $f, h, n, q$ are $n \times n$ 
matrices. Then the adjoint representation of the basis of the 3-Lie algebra $\mathcal{D}$ has the following form:

$$
\begin{aligned}
\left(\mathcal{Y}^{i j}\right)_{d}^{c} & =\left(\begin{array}{cccc}
0 & b_{1} & 0 & 0 \\
e_{1} & 0 & 0 & 0 \\
0 & 0 & 0 & l_{1} \\
0 & 0 & p_{1} & 0
\end{array}\right), \\
\left(\mathcal{Y}^{\tilde{i}}\right)_{d}^{c} & =\left(\begin{array}{cccc}
0 & b_{2} & 0 & 0 \\
e_{2} & 0 & 0 & 0 \\
0 & 0 & 0 & l_{2} \\
0 & 0 & p_{2} & 0
\end{array}\right), \\
\left(\mathcal{Y}^{+i}\right)_{d}^{c} & =\left(\begin{array}{llll}
0 & 0 & 0 & 0 \\
0 & f_{1} & 0 & 0 \\
0 & 0 & 0 & 0 \\
0 & 0 & 0 & q_{1}
\end{array}\right), \\
\left(\mathcal{Y}^{\tilde{+} \tilde{i}}\right)_{d}^{c}= & \left(\begin{array}{llll}
0 & 0 & 0 & 0 \\
0 & f_{2} & 0 & 0 \\
0 & 0 & 0 & 0 \\
0 & 0 & 0 & q_{2}
\end{array}\right),
\end{aligned}
$$

and we have $\mathcal{Y}^{-i}=0, \mathcal{Y}^{\tilde{-} \tilde{i}}=0, \mathcal{Y}^{-A}=0, \mathcal{Y}^{\tilde{-A}}=0$, where

$$
\begin{aligned}
& b_{1}=\left(\begin{array}{ccc}
0 & \cdots & 0 \\
f_{1}^{i j} & \cdots & f_{n}^{i j}
\end{array}\right), \quad e_{1}=\left(\begin{array}{cc}
f^{i j 1} & 0 \\
\vdots & \vdots \\
f^{i j n} & 0
\end{array}\right), \\
& l_{1}=\left(\begin{array}{ccc}
f^{i j 1} & \cdots & f^{i j n} \\
0 & \cdots & 0
\end{array}\right), \quad p_{1}=\left(\begin{array}{cc}
0 & f_{1}^{i j} \\
\vdots & \vdots \\
0 & f_{n}^{i j}
\end{array}\right) \text {, } \\
& b_{2}=\left(\begin{array}{ccc}
\tilde{f}_{i j 1} & \cdots & \tilde{f}_{i j n} \\
0 & \cdots & 0
\end{array}\right), \quad e_{2}=\left(\begin{array}{cc}
0 & \tilde{f}_{i j}^{1} \\
\vdots & \vdots \\
0 & \tilde{f}_{i j}^{n}
\end{array}\right) \text {, } \\
& l_{2}=\left(\begin{array}{ccc}
0 & \cdots & 0 \\
\tilde{f}_{i j}^{1} & \cdots & \tilde{f}_{i j}^{n}
\end{array}\right), \quad p_{2}=\left(\begin{array}{cc}
\tilde{f}_{i j 1} & 0 \\
\vdots & \vdots \\
\tilde{f}_{i j n} & 0
\end{array}\right) \text {, } \\
& f_{1}=\left(\begin{array}{ccc}
f_{1}^{i 1} & \cdots & f_{n}^{i 1} \\
\vdots & \vdots & \vdots \\
f_{1}^{i n} & \cdots & f_{n}^{i n}
\end{array}\right), \quad q_{1}=\left(\begin{array}{ccc}
f_{1}^{i 1} & \cdots & f_{1}^{i n} \\
\vdots & \vdots & \vdots \\
f_{n}^{i 1} & \cdots & f_{n}^{i n}
\end{array}\right) \text {, } \\
& f_{2}=\left(\begin{array}{ccc}
\tilde{f}_{i 1}^{1} & \cdots & \tilde{f}_{i n}^{1} \\
\vdots & \vdots & \vdots \\
\tilde{f}_{i 1}^{n} & \cdots & \tilde{f}_{i n}^{n}
\end{array}\right), \quad q_{2}=\left(\begin{array}{ccc}
\tilde{f}_{i 1}^{1} & \cdots & \tilde{f}_{i 1}^{n} \\
\vdots & \vdots & \vdots \\
\tilde{f}_{i n}^{1} & \cdots & \tilde{f}_{i n}^{n}
\end{array}\right) \text {, }
\end{aligned}
$$

where $f_{k}^{i j}=-\left(\mathcal{X}^{i}\right)_{k}^{j}$ and $\tilde{f}_{i j}^{k}=-\left(\tilde{\mathcal{X}}_{i}\right)_{j}^{k}$. The nondegenerate ad-invariant inner product for Lie algebras is the result of applying the trace of the bilinear product in the adjoint representation [47]. However, if the Lie algebra is non-semisimple, the current method is not useful. Obtaining the nondegenerate ad-invariant metric for these algebras results from solving the following equation:

$f_{D}^{A B} G^{C D}=-f_{D}^{A C} G^{D B}$.

Now, we generalize the above result for the 3-Lie algebra $\mathcal{D}$ and choose the basis of $\mathcal{D}=\mathcal{A} \oplus \mathcal{A}^{*}$ as $T^{A}\left(\left\{T^{-}, T^{+}, T^{i}\right.\right.$, $\left.\left.\tilde{T}_{-}, \tilde{T}_{+}, \tilde{T}_{i}\right\}\right)=\left\{T^{-}, T^{+}, T^{i}, T^{\tilde{\mathcal{}}}, T^{\tilde{+}}, T^{\tilde{i}}\right\}$, to obtain the inner product as follows:

$$
\begin{aligned}
\left\langle T^{A},\left[T^{B}, T^{C}, T^{D}\right]_{\mathcal{D}}\right\rangle & =-\left\langle\left[T^{A}, T^{B}, T^{C}\right]_{\mathcal{D}}, T^{D}\right\rangle \\
F_{E}^{B C D} G^{E A} & =-F_{E}^{A B C} G^{E D} .
\end{aligned}
$$

By choosing $\left(\mathcal{Y}^{A B}\right)_{D}^{C}=F_{D}^{A B C},(72)$ means that $\mathcal{Y}^{A B} G$ must be antisymmetric. We have shown their matrix representations as follows and have concluded that the metric must have the following form:

$G=\left(\begin{array}{cccc}a & 0 & b & 0 \\ 0 & 0 & 0 & h \\ i & 0 & k & 0 \\ 0 & n & 0 & 0\end{array}\right)$,

where

$$
\begin{array}{ll}
a=\left(\begin{array}{cc}
0 & g_{11} \\
g_{12} & 0
\end{array}\right), & b=\left(\begin{array}{cc}
0 & g_{21} \\
g_{22} & 0
\end{array}\right), \\
i=\left(\begin{array}{cc}
0 & g_{31} \\
g_{32} & 0
\end{array}\right), & k=\left(\begin{array}{cc}
0 & g_{41} \\
g_{42} & 0
\end{array}\right),
\end{array}
$$

in which the matrices $h$ and $n$ are arbitrary matrices.

\section{References}

1. E. Cremmer, B. Julia, J. Scherk, Supergravity theory in elevendimensions. Phys. Lett. B 76, 409 (1978)

2. E. Bergshoeff, E. Sezgin, P. Townsend, Supermembranes and eleven-dimensional supergravity. Phys. Lett. B 189, 75 (1987)

3. P.K. Townsend, D-branes from M-branes. Phys. Lett. B 373, 68 (1996). arXiv: hep-th/9601003

4. C. Schmidhuber, D-brane actions. Nucl. Phys. B 467, 146 (1996). arXiv: hep-th/9512062

5. D.S. Berman, M-theory branes and their interactions. Phys. Rept. 456, 89-126 (2008). arXiv: 0710.1707

6. J. Bagger, N. Lambert, Modeling multiple M2s. Phys. Rev. D 75, 045020 (2007). arXiv: hep-th/0611108

7. J. Bagger, N. Lambert, Gauge symmetry and supersymmetry of multiple M2-branes. Phys. Rev. D 77, 065008 (2008). arXiv:0711.0955

8. J. Bagger, N. Lambert, Comments on multiple M2-branes. JHEP 02, 105 (2008). arXiv:0712.3738

9. A. Gustavsson, Algebraic structures on parallel M2-branes. Nucl. Phys. B 811, 66 (2009). arXiv:0709.1260

10. O. Aharony, O. Bergman, D.L. Jafferis, J. Maldacena, $\mathrm{N}=6$ superconformal Chern-Simons-matter theories, M2-branes and their gravity duals. JHEP 10, 091 (2008). arXiv:0806.1218

11. J. Bagger, N. Lambert, Three-algebras and $\mathrm{N}=6$ Chern-Simons gauge theories. Phys. Rev. D 79, 025002 (2009). arXiv:0807.0163

12. A. Basu, J.A. Harvey, The M2-M5 brane system and a generalized Nahms equation. Nucl. Phys. B 713, 136 (2005). arXiv:hep-th/0412310 
13. W. Nahm, A simple formalism for the BPS monopole. Phys. Lett. B 90, 413 (1980)

14. M. Van Raamsdonk, Comments on the Bagger-Lambert theory and multiple M2- branes. JHEP 05, 105 (2008). arXiv:0803.3803

15. B. Ezhuthachan, S. Mukhi, C. Papageorgakis, D2 to D2. JHEP 0807, 041 (2008). arXiv:0806.1639

16. S. Mukhi, C. Papageorgakis, M2 to D2. JHEP 05, 085 (2008). arXiv:0803.3218v2

17. A. Sh Banerjee, A. Sen, Interpreting the M2-brane Action. Mod. Phys. Lett. 24, 721 (2009). arXiv:0805.3930

18. P.-M. Ho, Y. Imamura, Y. Matsuo, M2 to D2 revisited. JHEP 07, 003 (2008). arXiv:0805.1202

19. J. Gomis, G. Milanesi, J.G. Russo, Bagger-Lambert theory for general lie algebras. JHEP 06, 075 (2008). arXiv:0805.1012

20. Y. Pang, T. Wang, From N M2s to N D2s. Phys. Rev. D 78, 125007 (2008). arXiv:0807.1444

21. T. Li, Y. Liu, D. Xie, Multiple D2-brane action from M2-branes. Int. J. Mod. Phys. A 24, 3039 (2009). arXiv:0807.1183

22. P. de Medeiros, J.M. Figueroa-OFarrill, E. Mendez-Escobar, Metric Lie 3-algebras in Bagger-Lambert theory. JHEP 0808, 045 (2008). arXiv:0806.3242

23. P. Richmond, Multiple M-branes and 3-algebras, PHD thesis. arXiv: 1211.6930

24. A. Rezaei-Aghdam, L. Sedghi-Ghadim, 3-Libniz bialgebras (3-Lie bialgebra). arXiv: 1604.04475

25. V.G. Drinfeld, in Quantum groups. Proceedings of the international congress of mathematicians, (Berkeley, 1986), p. 798

26. I. Vaisman, Lectures on the Geometry of Poisson Manifolds, Progress in Mathe- matics, Birkhauser Basel, Vol 118 (1994)

27. Y. K. Schwarzbach, Lie bialgebras, Poisson-Lie groups and dressing transformations, Integrability of nonlinear systems. Lecture notes in physics 038, 2nd edn. (Springer-Verlag, 2004), p. 107

28. S.E. Parkhomenko, Extended superconformal current-algebras and finite-dimensional Manin triples. Sov. Phys. JETP 75(1), 1 (1992)

29. S. Parkhomenko, Quasi Frobennius Lie algebras construction of $N=4$ superconformal field theory. Mod. Phys. lett. A 11, 445 (1996)

30. U. Lindström, M. Zabzine, D-branes in $\mathrm{N}=2$ WZW models. Phys. Lett. B 560, 108 (2003). arXiv: hep-th/0212042
31. M. Aali-Javanangrouh, A. Rezaei-Aghdam, Algebraic Structures of $\mathrm{N}=(4,4)$ and $\mathrm{N}=(8,8)$ SUSY Sigma Models on Lie groups and SUSY WZW Models. arXiv:1402.5600v1

32. V. Filippov, n-Lie algebras. Sibirsk. Mat. Zh. 26, 126140 (1985)

33. Y. Nambu, Generalized Hamiltonian mechanics. Phys. Rev. D 7, 17405 (1973)

34. G. Sarkissian, On D-branes in the Nappi-Witten and GMM gauged WZW models. JHEP 0301, 059 (2003). arXiv: hep-th/0211163

35. P. Bordalo, S. Ribault, C. Schweigert, Flux stabilization in compact groups. JHEP 0110, 036 (2001). arXiv: hep-th/0108201

36. S. Stanciu, A note on D-branes in group manifolds: flux quantization and D0-charge. JHEP 0010, 015 (2000). arXiv: hep-th/0006145

37. L. Takhtajan, On foundations of generalized Nambu mechanics. Commun. Math. Phys. 160, 295 (1994)

38. Miguel A. Bandres, Arthur E. Lipstein, John H. Schwarz, $\mathrm{N}=8$ Superconformal Chern-Simons Theories. JHEP 0805, 025 (2008). arXiv:0803.3242

39. A. Rezaei-Aghdam, M. Hemmati, A.R. Rastkar, Classification of real three-dimensional Lie bialgebras and their Poisson-Lie groups, J. Phys. A: Math. Gen. 38, 3981 (2005). arXiv: math-ph/0412092

40. L. Takhtajan, Deformation quantization and Nambu mechanics. Commun. Math. Phys. 183, 1 (1997)

41. M. Aali-Javanangrouh, A. Rezaei-aghdam, From Basu-Harvey to Nahm equation via 3-Lie bialgebra. arXiv:1604.05181

42. D. Tong, Lectures on String Theory (February 2012)

43. J. Polchinski, TASI lectures on D-branes. arXiv:hep-th/9611050

44. J. Polchinski, Dirichlet Branes and Ramond-Ramond charges. Phys. Rev. Lett. 75, 4724 (1995). arXiv:hep-th/9510017

45. R.G. Leigh, Dirac-Born-Infeld action from the Dirichlet sigma model. Mod. Phys. Lett. A 4, 2767 (1989)

46. C. Nappi, E. Witten, A WZW model based on a non-semi-simple group. Phys. Rev. Lett. 71, 3751-3753 (1993)

47. M.A. Jafarizadeh, A. Rezaei-Aghdam, Poisson-Lie T-duality and Bianchi Type algebras. Phys. Lett. B 458, 477 (1999). arXiv: hep-th/9903152 